\title{
Myocardin-related transcription factor A nuclear translocation contributes to mechanical overload-induced nucleus pulposus fibrosis in rats with intervertebral disc degeneration
}

\author{
MENG KONG $^{1 *}$, YIRAN ZHANG $^{1,2^{*}}$, MENGXIONG SONG $^{1}$, WENBIN CONG $^{3}$, \\ CHANGTONG GAO ${ }^{4}$, JIAJUN ZHANG ${ }^{1}$, SHUO HAN ${ }^{1}$, QIHAO TU ${ }^{1}$ and XUEXIAO MA ${ }^{1,2}$ \\ ${ }^{1}$ Department of Spinal Surgery, The Affiliated Hospital of Qingdao University; \\ ${ }^{2}$ Institute of Traumatic Orthopedics in Shandong Province; ${ }^{3}$ Department of Radiology, \\ The Affiliated Hospital of Qingdao University; ${ }^{4}$ Minimally Invasive Interventional Therapy Center, \\ Qingdao Municipal Hospital, Qing'dao, Shandong 266000, P.R. China
}

Received December 6, 2020; Accepted April 16, 2021

DOI: 10.3892/ijmm.2021.4956

\begin{abstract}
Previous studies have reported that the Ras homolog family member A (RhoA)/myocardin-related transcription factor A (MRTF-A) nuclear translocation axis positively regulates fibrogenesis induced by mechanical forces in various organ systems. The aim of the present study was to determine whether this signaling pathway was involved in the pathogenesis of nucleus pulposus (NP) fibrosis induced by mechanical overload during the progression of intervertebral disc degeneration (IVDD) and to confirm the alleviating effect of an MRTF-A inhibitor in the treatment of IVDD. NP cells (NPCs) were cultured on substrates of different stiffness (2.9 and $41.7 \mathrm{KPa}$ ), which mimicked normal and overloaded microenvironments, and were treated with an inhibitor of MRTF-A nuclear import, CCG-1423. In addition, bipedal rats were established by clipping the forelimbs of rats at 1 month and gradually elevating the feeding trough, and in order to establish a long-term overload-induced model of IVDD, and their intervertebral discs were injected with CCG-1423 in situ. Cell viability was determined by Cell Counting Kit- 8 assay, and protein expression was determined by western blotting, immunofluorescence and immunohistochemical staining. The results demonstrated that the viability of NPCs was not affected by the application of force or the inhibitor. In NPCs cultured on stiff matrices, MRTF-A was mostly localized in the nucleus, and the expression levels of fibrotic proteins,
\end{abstract}

Correspondence to: Dr Xuexiao Ma, Department of Spinal Surgery, The Affiliated Hospital of Qingdao University, 59 Hai Er Road, Qing'dao, Shandong 266000, P.R. China

E-mail: maxuexiaospinal@163.com

*Contributed equally

Key words: mechanical overload, intervertebral disc degeneration, actin, myocardin-related transcription factor $\mathrm{A}$, fibrosis, rat including type I collagen, connective tissue growth factor and $\alpha$-smooth muscle cell actin, were upregulated compared with those in NPCs cultured on soft matrices. The levels of these proteins were reduced by CCG-1423 treatment. In rats, 6 months of upright posture activated MRTF-A nuclear-cytoplasmic trafficking and fibrogenesis in the NP and induced IVDD; these effects were alleviated by CCG-1423 treatment. In conclusion, the results of the present study demonstrated that the RhoA/MRTF-A translocation pathway may promote mechanical overload-induced fibrogenic activity in NP tissue and partially elucidated the molecular mechanisms underlying the occurrence of IVDD.

\section{Introduction}

Intervertebral disc (IVD) degeneration (IVDD) has long been considered to contribute to lower back pain, which has a prevalence ranging between 1.4 and $20.0 \%$ (1) and has been one of the three leading causes of non-fatal health loss for nearly three decades (2), leading to a considerable economic burden on health care systems (3). Therefore, novel treatments that effectively prevent and treat IVDD are urgently needed. Understanding the mechanism underlying the pathogenesis of IVDD is of great significance for developing effective therapeutics to treat IVDD.

Epidemiological studies have reported that excessive mechanical stress is one of the major factors that induce IVDD, that the IVD is always affected by stress even in a resting state, and that overloaded compressive force accelerates the initiation and progression of IVDD $(4,5)$.

The IVD contains a nucleus pulposus (NP) core, surrounded by an outer ring of the annulus fibrosus (AF) between two cartilaginous endplates. A previous study has demonstrated that NP fibrosis triggers and serves a prominent role in the progression of IVDD (6). In addition to the degradation and gradual loss of aggrecan and type II collagen, and the enhanced production of fibrotic proteins, the progression of tissue fibrosis is characterized by the excessive remodeling and abnormal deposition of extracellular matrix (ECM) components and the subsequent 
accentuated distortion of the architecture, which has been observed in the NP of degenerative discs $(7,8)$.

Previous studies have demonstrated that the Rho GTPase family and its downstream signaling pathways are involved in the basic cellular regulatory mechanisms associated with biological activities and diseases, including proliferation, differentiation, migration and gene expression $(9,10)$. The role of the mechanical stress/Ras homolog family member A (RhoA)/myocardin-related transcription factor A (MRTF-A)-mediated pathway in cellular mechanical stress responses and the fibrotic process of a number of organ systems, including the heart, lung, eye and kidney, has been previously established (11-15). Mechanical stimulus-initiated signals derived from the ECM are detected by cognate transmembrane receptor proteins termed integrins, which are the main structure-organizing components of focal adhesions; subsequently, RhoA signaling is activated through selective Rho guanine nucleotide exchange factors (9). Consequently, monomeric globular actin (G-actin) subunits in the cytoplasm assemble into long filamentous F-actin polymers. Following RhoA-induced actin polymerization, MRTF-A is released from $\mathrm{G}$-actin and translocated to the nucleus, where it acts as a transcriptional cofactor for serum response factor (SRF) by binding to the $\mathrm{CArG}$ regulatory sequence $[\mathrm{CC}(\mathrm{A} / \mathrm{T}) 6 \mathrm{GG}]$ in the promoter regions of its target genes, resulting in the increased expression levels of type I collagen, connective tissue growth factor (CTGF), smooth muscle cell actin (SMA) and other fibrosis-related factors $(9,16)$. To the best of our knowledge, no relevant studies have been conducted to determine whether this molecular mechanism serves a dominant role in the process of the fibrotic changes observed in NP cell types to date.

A previous study has demonstrated that CCG-1423 is a small molecule inhibitor that specifically binds to the $\mathrm{N}$-terminal basic domain (which acts as a functional nuclear localization signal) of MRTF-A and inhibits the nuclear import of MRTF-A (17). Therefore, the present study aimed to determine the role of the RhoA/MRTF-A signaling pathway in mechanically induced NP fibrosis in rats in vivo and in vitro, and to investigated whether pharmacological inhibition by the MRTF-A chemical inhibitor CCG-1423 may alleviate NP fibrogenesis.

\section{Materials and methods}

Materials. The primary antibodies against RhoA (cat. no. A15641), MRTF-A (cat.no. A8504), $\alpha$-SMA (cat.no. A7248), CTGF (cat. no. A11067), collagen type I (cat. no. A1352) and II (cat. no. A1560) were purchased from ABclonal Biotech Co., Ltd. The anti-F-actin antibody (cat. no. ab205) was purchased from Abcam, the anti- $\beta$-actin antibody (cat. no. AF5003) was purchased from Beyotime Institute of Biotechnology, and CCG-1423 was purchased from Cayman Chemical Company.

Ex vivo rat NP explant preparation and treatment. The present study was approved by the Laboratory Animal Care and Use Committee of the Affiliated Hospital of Qingdao University (Qing'dao, China; approval no. QYFYWZLL 26037).

Cell culture. NP explants were obtained from one female Sprague-Dawley rat (age, 1 month) as previously described (18).
In brief, the rat was euthanized by intraperitoneal injection of sodium pentobarbital (100 $\mathrm{mg} / \mathrm{kg}$ ); the spinal column was removed under aseptic conditions, and the gel-like NP was separated from the AF. The NP tissues were digested with $0.01 \%$ trypsin (HyClone; Cytiva) for $\sim 30 \mathrm{~min}$ at $37^{\circ} \mathrm{C}$, followed by treatment with $0.125 \%$ collagenase II (MilliporeSigma) for $\sim 4 \mathrm{~h}$. The digested tissue was filtered using a $100-\mu \mathrm{m}$ cell strainer and washed twice with phosphate-buffered saline (PBS; Gibco; Thermo Fisher Scientific, Inc.). The isolated NPCs were maintained in Dulbecco's modified Eagle's medium (DMEM, Gibco; Thermo Fisher Scientific, Inc.) supplemented with $10 \%$ fetal bovine serum (FBS; Gibco; Thermo Fisher Scientific, Inc.) and $1 \%$ penicillin/streptomycin under hypoxic conditions with $5 \% \mathrm{O}_{2}$ and $5 \% \mathrm{CO}_{2}$ at $37^{\circ} \mathrm{C}$, and the culture media were exchanged every 3 days. All experiments were completed with low-passage $(<3)$ cells cultured in monolayers.

Polyacrylamide (PA) substrate preparation. PA hydrogels are a well-established matrix substrate system used for matrix stiffness assays (11). PA gels (PAGs) with defined mechanical stiffness levels were prepared by mixing polymerizing acrylamide (8\% final concentration, prepared from a $40 \%$ stock solution; Bio-Rad Laboratories, Inc.) with two concentrations of bis-acrylamide (0.048 and $0.48 \%$, prepared from $2 \%$ stock solution; Bio-Rad Laboratories, Inc.) to create substrates of two different stiffnesses (19). The procedures for preparing the substrates and determining the modulus values were adopted from previous studies $(20,21)$. Briefly, the gels were prepared between two glass coverslips respectively coated with 3-aminopropyl trimethoxy silane and octadecyl-trichlorosilane. The thickness of the gel was controlled by adjusting the volume of the solution placed between the coverslips. Following gelation, the nonadherent plate was removed. Fibronectin $(10 \mu \mathrm{g} / \mathrm{ml}$; MilliporeSigma), which is an abundant ECM ligand in the NP to which NPCs adhere (20), was added dropwise to the surface of the treated gel using sulfo-SANPAH-based conjugation. Before seeding the cells, the polyacrylamide gels were washed twice with precooled sterile HEPES ( $\mathrm{pH} \mathrm{8.5)} \mathrm{and} \mathrm{equilibrated}$ with media for $1 \mathrm{~h}$ at room temperature. To prevent the stimulation of SRF signaling by serum and the potential paracrine signaling induced by cell adherence to the surrounding plastic coverslip, prior to transferring the coverslip and hydrogel to a new 6-well plate containing minimal FBS $(0.5 \%)$, the NPCs were allowed to attach to the prepared matrix for $2 \mathrm{~h}$.

For the inhibitor experiments, CCG-1423 was diluted in vehicle $(0.1 \%$ DMSO) to obtain a final concentration of $10 \mu \mathrm{M}$ according to a previous study (22). NP cells were seeded on either stiff or soft substrate in reduced-serum (0.5\% FBS) DMEM to reduce the serum-induced activation of gene expression (23). According to the manufacturer's instructions, $10 \mu \mathrm{M}$ CCG-1423 and an equivalent volume of $0.1 \%$ DMSO were added to the cells and cultured for $72 \mathrm{~h}$ at $37^{\circ} \mathrm{C}$. A total of four experimental groups were established in the subsequent experiments: i) Soft, NPCs cultured on a soft substrate without intervention; ii) stiff, NPCs cultured on a stiff substrate without intervention; iii) soft + CCG-1423, NPCs cultured on a soft substrate with CCG-1423; and iv) stiff + CCG-1423, NPCs cultured on a stiff substrate with CCG-1423. Subsequently, the cytotoxicity of the inhibitor and solvent was assessed using the Cell Counting Kit-8 (CCK-8) assay (Beyotime Institute of 
Biotechnology) by adding $10 \%$ CCK- 8 solution to the cells for $2 \mathrm{~h}$ at $37^{\circ} \mathrm{C}$, and the viability of the cells in the four groups was determined by measuring the optical density value at $450 \mathrm{~nm}$. Images of the cells were captured under an inverted Olympus BX51 light microscope (magnification, x20; Olympus Corporation) for identification, and the cells harvested for protein detection or fixed for immunofluorescence staining and confocal microscopy.

Immunofluorescence imaging. To visualize MRTF-A, following NPC incubation and treatment, the gels were washed with PBS, and the NPCs were fixed with $4 \%$ paraformaldehyde at room temperature for $10 \mathrm{~min}$, followed by permeabilization with $0.2 \%$ Triton $\mathrm{X}-100$ in PBS for $15 \mathrm{~min}$ at room temperature. Subsequently, the gels were washed 3 times with precooled PBS and blocked with PBS containing $1 \%$ BSA (Beyotime Institute of Biotechnology). The gels were incubated with rabbit anti-MRTF-A (1:200) and anti-F-actin (1:200) primary antibodies at room temperature for $2 \mathrm{~h}$. Following washing three times with PBS, the NPCs were incubated with FITC-conjugated anti-rabbit IgG (cat. no. F9887, MilliporeSigma) and TRITC-conjugated anti-mouse IgG secondary antibodies (cat. no. T5393, MilliporeSigma) diluted 1:1,000 in PBS/0.1\% Triton for $30 \mathrm{~min}$ at room temperature. After three times of washes with PBS, the cell nuclei were counterstained with 4',6-diamidino-2-phenylindole (DAPI; MilliporeSigma). The nuclear localization of MRTF-A and F-actin was analyzed by a Nikon A1 confocal microscope (magnification, x20; Nikon Corporation) and quantified by ImageJ software (version $1.52 \mathrm{v}$; National Institutes of Health) to determine the integral optical density. The analyses were performed in a blinded manner.

Western blotting. The protein concentrations of the cell lysates obtained using RIPA lysis buffer (Beijing Solarbio Science \& Technology Co., Ltd.) were detected with the BCA protein assay kit (Beijing Solarbio Science \& Technology Co., Ltd). Then, samples with equal amounts of proteins (30 $\mu \mathrm{g} / \mathrm{lane})$ were resolved by $10 \%$ SDS-polyacrylamide gel electrophoresis, electrotransferred onto nitrocellulose membranes (MilliporeSigma), blocked with 5\% BSA at room temperature for $1 \mathrm{~h}$ and incubated with primary antibodies against RhoA $(1: 1,000)$, F-actin $(1: 1,000)$, type I collagen $(1: 5,000)$, type II collagen $(1: 5,000), \beta$-actin $(1: 1,000), \alpha$-SMA $(1: 1,000)$ and CTGF $(1: 1,000)$ overnight at $4^{\circ} \mathrm{C}$. Subsequently, the blots were washed with Tris-buffered saline with $1 \%$ Tween-20 and incubated with appropriate anti-rabbit (cat. no. ZB-2301) or mouse (cat. no. ZB-2305) IgG HRP-conjugated antibodies (both 1;3,000; OriGene Technologies, Inc.) at $37^{\circ} \mathrm{C}$ for $2 \mathrm{~h}$. The membranes were developed with a chemiluminescence reagent (Thermo Fisher Scientific, Inc.) using the ChemiDoc ${ }^{\mathrm{TM}}$ MP Imaging System (Bio-Rad Laboratories, Inc.), and densitometry was performed using ImageJ software.

IVDD animal model and surgical techniques. A total of 60 healthy female Sprague-Dawley rats (Charles River Laboratories, Inc.; specific pathogen-free; initial weight, 80-100 g) aged one month were provided by the Experimental Animal Center of The School of Medicine at Qingdao University. The animals were housed in an environmentally controlled room with a 12-h light cycle. Initially, two experimental groups were established: The intact control group $(n=15)$ and the IVDD model group $(n=45)$. Establishment of the animal model was conducted as previously described $(24,25)$. Briefly, the animals were anesthetized with sodium pentobarbital (40 mg/kg, intraperitoneally) and aseptically treated during the experiments. Transverse circular incisions were made over the axilla of the bilateral forelimbs of each rat. Both brachial plexus rhizotomy as well as distal skeleton and musculature amputation procedures were performed. The height of the food and water was gradually increased from 15 (standard height) to $30 \mathrm{~cm}$ (maximum height) as the length and weight of the rats increased to induce bipedalism. Within 6 months, this procedure led to the development of IVDD. Age-matched untreated rats served as the intact controls.

CCG-1423 treatment. To assess the therapeutic effects of CCG-1423 in vivo, at 3 months post-surgery, the animals in the IVDD group were randomly divided into three subgroups: i) IVDD model, bipedal rats without treatment; ii) CCG-1423 treatment, bipedal rats injected with CCG-1423 dissolved in DMSO; and iii) vehicle (DMSO), bipedal rats injected with DMSO alone. Through a retroperitoneal approach and with the exposure of the lumbar disc, $2 \mu$ l CCG-1423 (effective dose of $5 \mu \mathrm{g}$ dissolved in DMSO) or the same volume of DMSO was injected into the NP of L4/5 IVD using a microliter syringe (5 $\mu$; Shanghai Gaoge Industrial and Trading CO., Ltd.) attached to a $31-\mathrm{G}$ needle.

MRI and data processing. At 3 months post-injection, the rats were sedated with $10 \%$ chloral hydrate $(300 \mathrm{mg} / \mathrm{kg}$, intraperitoneally) for the MRI scans. The lumbar spines of the rats were assessed in a 3.0 T Varian MR scanner (MAGNETOM Skyra; Siemens AG) to obtain T2-weighted images (Water: SAG IDEAL fat-suppression sequence; repletion time, 3,000 ms; echo time, $80 \mathrm{~ms}$; field of view, $150 \times 150 \mathrm{~mm}$; slice thickness, $1.2 \mathrm{~mm}$ ) in the midsagittal plane. None of the animals exhibited signs of peritonitis, pain or discomfort. According to the modified Thompson classification $(26,27)$, the discs in the T2-weighted images were scored from grade I to IV.

All the assessments and quantitative data collection were performed by three independent, blinded observers and calculated as the mean of the three evaluations.

Histological analyses. Following the MRI scans, the rats were euthanized using the aforementioned method, and histological examinations were performed on samples from the four groups. The L4/5 IVD along with the adjacent vertebrae dissected from the four groups ( $\mathrm{n} \geq 5$ per group) were fixed in $10 \%$ buffered formalin ( $\mathrm{pH} \mathrm{7.4)} \mathrm{for} 24 \mathrm{~h}$, decalcified in a $10 \%$ EDTA solution for 3 weeks, embedded in paraffin and sectioned transversely at $3-\mu \mathrm{m}$ thickness with a microtome. The sections were stained with hematoxylin and eosin (H\&E). All procedures were conducted at room temperature. The cellularity and morphology of the NPs were independently examined by two experienced histology researchers in a blinded manner using a panoramic scanning microscope (magnification, x20; Pannoramic DESK, P-MIDI, P250, P1000; 3DHISTECH, Ltd.) with the accompanying CaseViewer software (version 2.3; 3DHISTECH, Ltd.). 
A

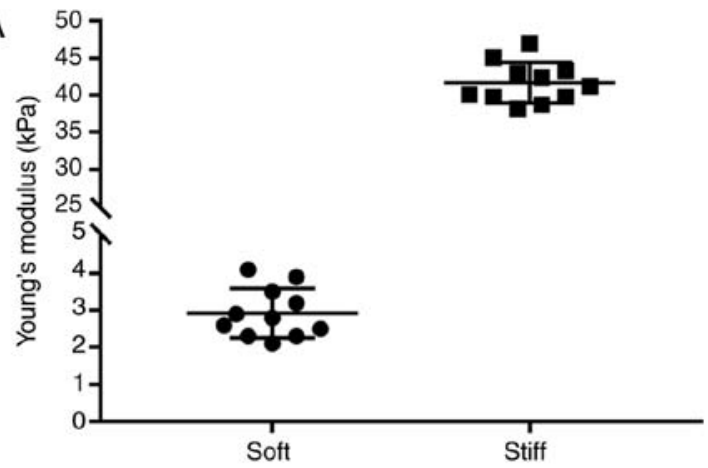

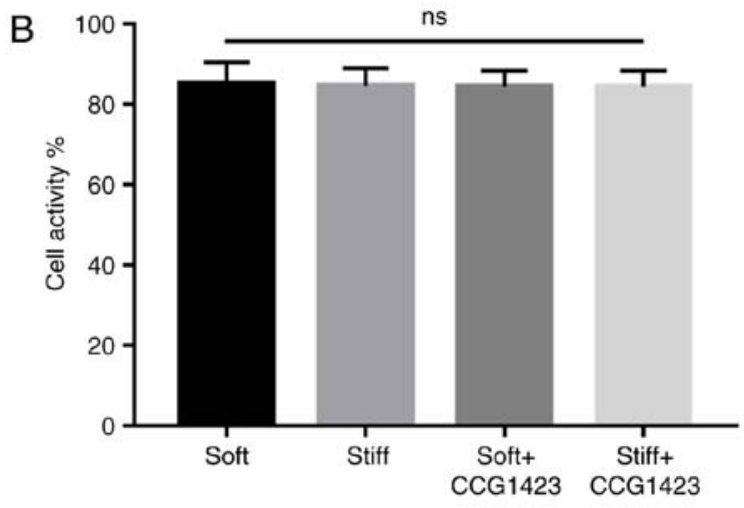

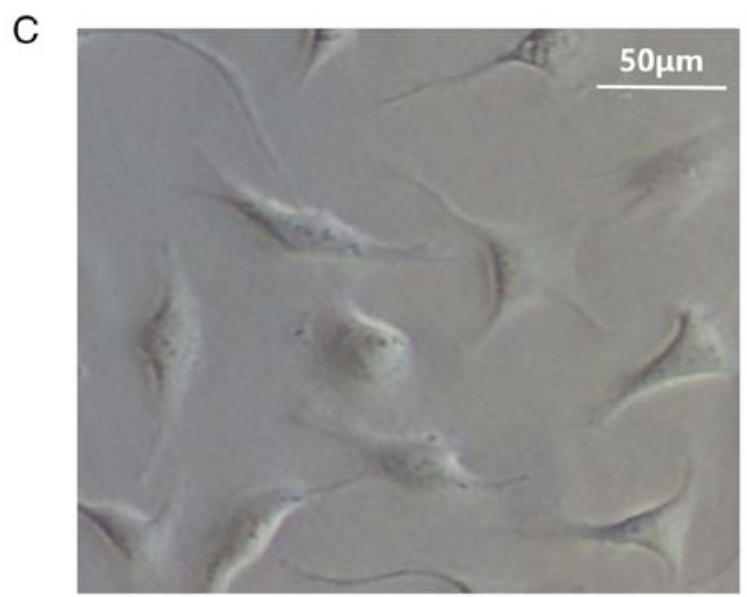

Figure 1. Effects of polyacrylamide gel stiffness on cell viability. (A) The stiffness/elasticity of soft and stiff polyacrylamide hydrogels were determined by dynamic fatigue testing machine in five locations. Young's modulus of each individual location of the gels and the mean values of the soft and stiff gel stiffness \pm SD are presented. (B) The effects of substrate siffness and 72-h CCG-1423 treatment on nucleus puplosus cell viability. (C) Representative image of NPCs captured under light microscope. ns, not significant. NPCs, nucleus pulposus cells.

The disc degeneration scores in all groups were assessed and calculated according to a modified grading system as previously described $(28,29)$.

Immunohistochemical staining. Immunohistochemical staining was performed to examine the expression of MRTF-A (1:200), CTGF (1:100), $\alpha$-SMA (1:100) and type I collagen (1:200) in the tissues. Following deparaffinization, each specimen was incubated for $5 \mathrm{~min}$ at room temperature with $3 \%$ hydrogen peroxide to eliminate the endogenous peroxidase activity. Subsequently, $20 \%$ goat serum (OriGene Technologies, Inc.) was used to block the nonspecific protein binding sites. This step was followed by incubation with the corresponding antibodies at $4^{\circ} \mathrm{C}$ overnight. The following day, the tissue sections were incubated for $30 \mathrm{~min}$ with horseradish peroxidase-conjugated goat anti-rabbit IgG secondary antibodies (1:200; cat. no. ZB-2301; OriGene Technologies, Inc.) at $37^{\circ} \mathrm{C}$. The color was developed by incubation with chromogen 3,3'-diaminobenzidine (DAB) tetrahydrochloride, followed by counterstaining with hematoxylin for $3 \mathrm{~min}$ at room temperature. An Olympus IX71 light microscope was used to capture the images (magnification, x60) in a total of five randomly selected regions in each immunohistochemical section, and the positive cells were counted with Image J software. NPCs in which the nucleus was stained and the cytoplasm exhibited brown staining were considered to be positive for the target proteins.
Tissue immunofluorescence. The whole $\mathrm{L} 4 / 5$ disc along with the adjacent vertebrae of the remaining rats ( $\mathrm{n} \geq 5$ per group) were frozen and coronally sectioned into $5-\mu \mathrm{m}$ sections using a Leica CM1950 cryostat (Leica Microsystems GmbH); subsequently, the sections were stained as aforementioned.

Statistical analysis. All experiments were replicated three times. Quantitative data are presented as the mean \pm standard error. Statistical analysis was performed using GraphPad Prism 7 software (GraphPad Software, Inc.). Data were analyzed using one-way ANOVA with Tukey's multiple comparison test. $\mathrm{P}<0.05$ was considered to indicate a statistically significant difference.

\section{Results}

Stiffness activates RhoA in nucleus pulposus cells. Soft and stiff PAGs that mimic the physical properties of normal and overloaded microenvironments, respectively, were established for the in vitro experiments in the present study. Dynamic fatigue testing machine measurements demonstrated that the soft PAGs had a mean stiffness value (Young's modulus) of $2.9 \pm 0.2 \mathrm{kPa}$, whereas the stiff PAGs had a mean value of $41.7 \pm 0.8 \mathrm{kPa}$ (Fig. 1A).

Following seeding, within $72 \mathrm{~h}$, the NPCs gradually adhered to the bottom of the culture dish (Fig. 1C). The results of the CCK-8 assay revealed that the cell viability was comparable in the four groups, suggesting that neither stiff nor soft 
A
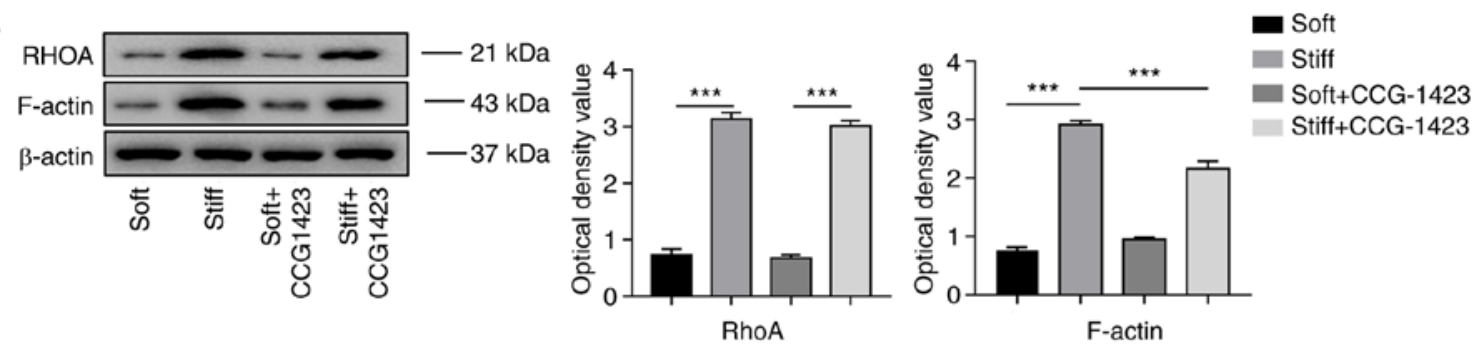

B

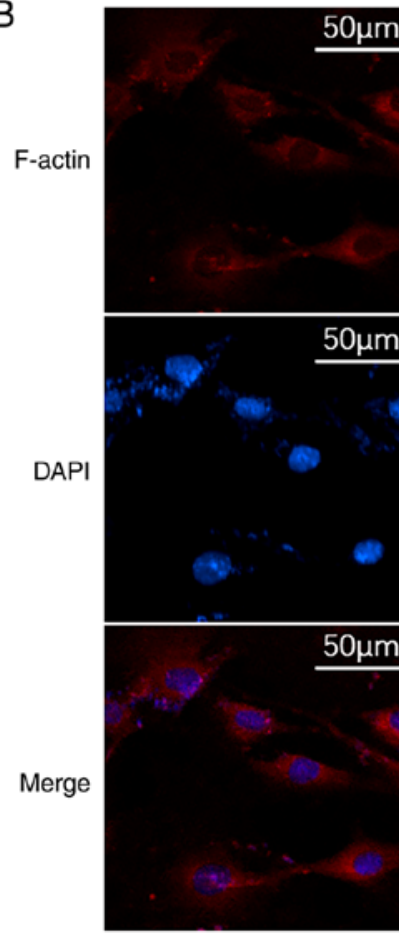

Soft

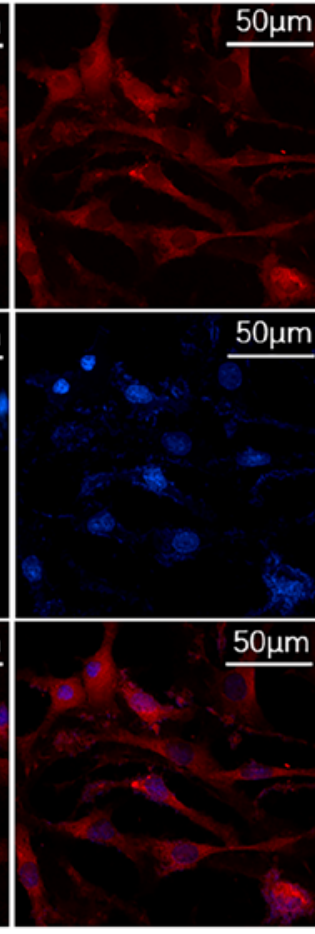

Stiff

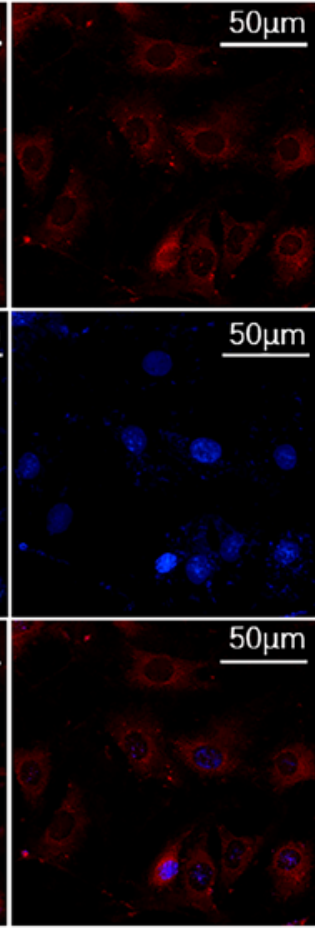

Soft+CCG1423

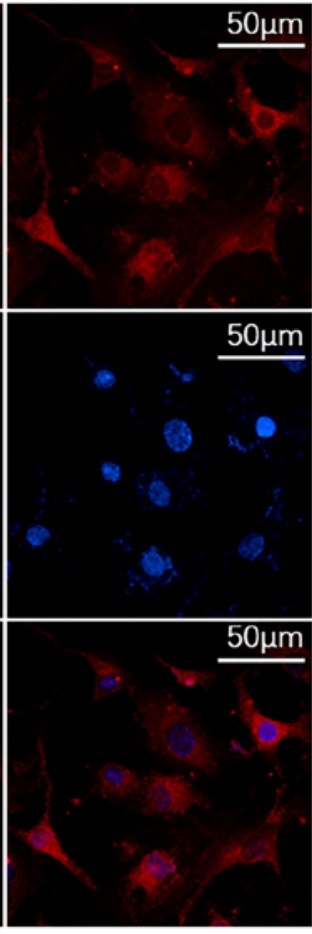

Stiff+CCG1423

Figure 2. Expression levels of RhoA and F-actin in NPCs. (A) Western blot analysis results demonstrated that the levels of RhoA and F-actin were higher in NPCs of the stiff group compared with those in the soft group, and CCG-1423 treatment reduced the levels of F-actin of NPCs cultured on a stiff matrix. No significant differences were observed in RhoA levels between cells in the stiff and stiff + CCG-1423 groups (B) F-actin staining (red) in NPCs in the soft, stiff, soft + CCG-1423 and stiff + CCG-1423 groups. NPCs with a spindle morphology were observed in the stiff group. Cell nuclei were visualized with DAPI (blue). ${ }^{* * * *} \mathrm{P}<0.001$. NPCs, nucleus pulposus cells; RhoA, Ras homolog family member A.

substrates exerted significant effects on the proliferation of NPCs in $72 \mathrm{~h}$ (Fig. 1B).

Under serum-free conditions, the western blotting results demonstrated that the protein levels of RhoA and F-actin were upregulated in the NPCs in the stiff group compared with those in the NPCs in the soft group (Fig. 2A). The results of the immunofluorescence analysis also confirmed that a stiff matrix induced changes in actin dynamics, as more intense F-actin staining was observed in the cytoplasm of rat NPCs cultured on a stiff matrix compared with that in the cytoplasm of rat NPCs cultured on a soft matrix (Fig. 2B).

Stiff matrix induces nuclear-cytoplasmic trafficking of $M R T F-A$ and expression of associated factors. The impact of mechanical cues on the subcellular localization of MRTF-A was further determined. Based on the localization of MRTF-A-GFP in fluorescence microscopy images, the NP cells were classified into two categories: Mainly nuclear or mainly cytoplasmic. MRTF-A was mainly located in the cytoplasm in $81 \%$ of the cells in the soft group, with a mean nuclear state of $13-25 \%$. As demonstrated by immunofluorescence, culture on a stiff matrix resulted in notable nuclear staining of MRTF-A, and the proportion of MRTF-A in the nuclei of the NPCs was significantly increased compared with that in the soft group (75\% nuclear and $25 \%$ cytoplasmic; Fig. 3 ).

Since high levels of $\alpha$-SMA and type I collagen are major characteristics of fibrogenic activation (30), the present study examined the discrepancy in their expression in NPCs cultured on soft and stiff matrices. As demonstrated by western blotting, the protein levels of targeted fibrogenic cytokines, such as type I collagen and CTGF, were nearly undetectable in the NPCs cultured on the soft substrate, whereas increasing matrix stiffness caused a significant increase in the levels of the two proteins. In addition, elevated $\alpha$-SMA and downregulated collagen II expression levels were also observed in the stiff matrix-cultured cells compared with those in the soft group (Fig. 4A).

Inhibition of MRTF-A alleviates the levels of fibrogenic factor expression in NP cells exposed to mechanical stress. Treatment with $10 \mu \mathrm{M}$ CCG-1423 for $72 \mathrm{~h}$ partially blocked the stiff matrix-induced nuclear accumulation of MRTF-A in the NP cells (Fig. 3). Consistently, the protein levels of F-actin 


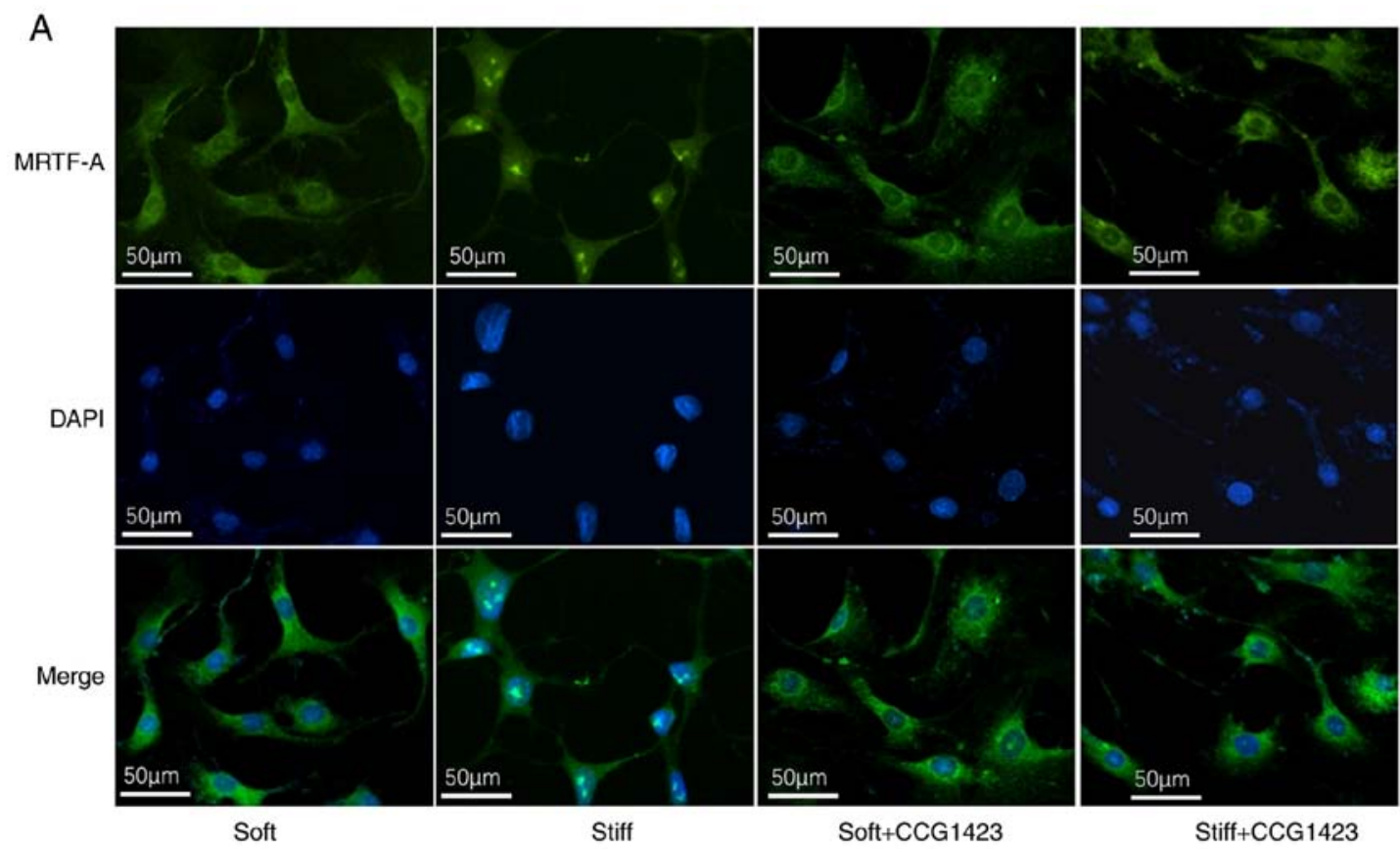

B

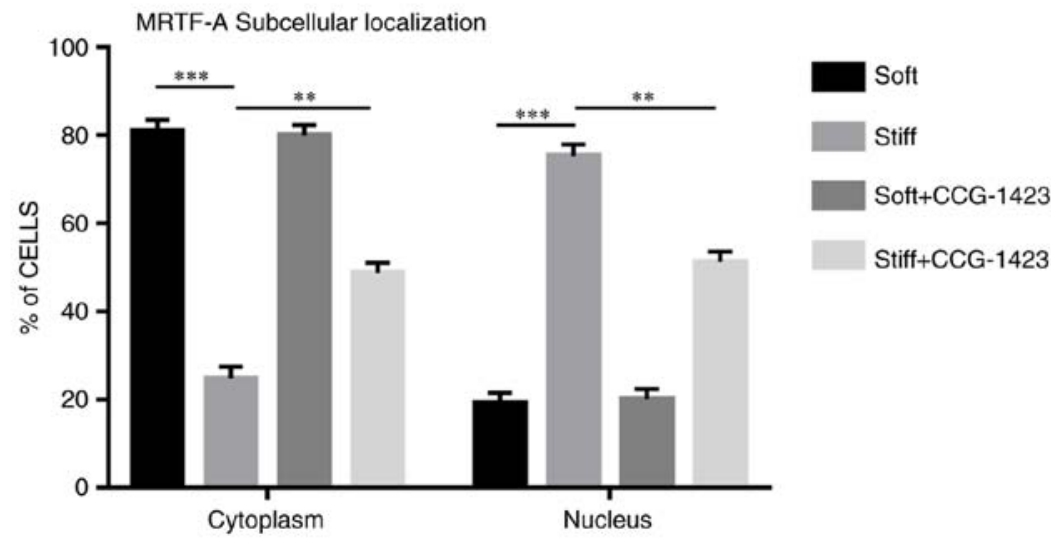

Figure 3. Immunofluorescence analysis of the effects of stiffness and CCG-1423 on MRTF-A subcellular localization in NPCs. (A) MRTF-A translocated to the nucleus in cells cultured on a stiff substrate. In CCG-1423-treated cells cultured on a stiff matrix, MRTF-A was retained mostly in the cytoplasm. Blue, DAPI; green, MRTF-A. (B) Percentage of cells in which MRTF-A was scored as predominantly cytoplasmic or predominantly following culture on a soft or stiff substrate and inhibitor treatment. Data are presented as the mean $\pm \mathrm{SEM} .{ }^{* *} \mathrm{P}<0.01$ and ${ }^{* * * *} \mathrm{P}<0.001$. MRTF-A, myocardin-related transcription factor A.

were reduced in the stiff + CCG-1423 compared with those in the stiff group (Fig. 2). However, no significant differences were observed in the levels of RhoA with or without CCG-1423 treatment in NPCs (Fig. 2A). Treatment with CCG-1423 significantly abrogated matrix stiffness-induced $\alpha$-SMA and type I collagen expression, as well as CTGF expression to levels below those observed in cells that received no treatment. These results suggested that the nuclear translocation of MRTF-A induced by a stiff matrix stimulated the expression of the SRF-induced proteins $\alpha$-SMA, type I collagen and CTGF. Another protein, type II collagen, exhibited an opposite trend compared with that of the three aforementioned markers (Fig. 4A).

Changes in the histopathology of intervertebral discs with increasing modeling time and CCG-1423 treatment. In the in vivo experiments, the present study first aimed to assess the progression of IVDD. Fig. 4B demonstrates representative T2-weighted midsagittal images of the approached rat lumbar disc acquired by MRI. At 6 months after the initial modeling surgery, all bipedal rats exhibited weaker signal densities in the L4/5 discs compared with those in the intact controls, and the MRI images of the NP in the CCG-1423-treated group revealed stronger signal intensities compared with those in the IVDD model and DMSO groups. The perturbed disc degeneration grades were determined by the modified Thompson grading evaluation revealed that the rats in the three IVDD groups exhibited higher grades compared with those in the control group. The CCG-1423-treated group exhibited lower grades compared with those in the IVDD or DMSO groups, suggesting that CCG-1423 treatment inhibited the reduction in the MRI signal intensity in the IVDD model discs.

The fibrotic changes in the NP were subsequently verified, and the results demonstrated that long-term (6 months) upright posture resulted in notable NP fibrosis. As demonstrated by the H\&E staining (Fig. 5A), in the control group, the NP remained relatively normal cellularity, and large numbers of vacuolated notochordal cells and round chondrocyte-like cells were distributed between 
A

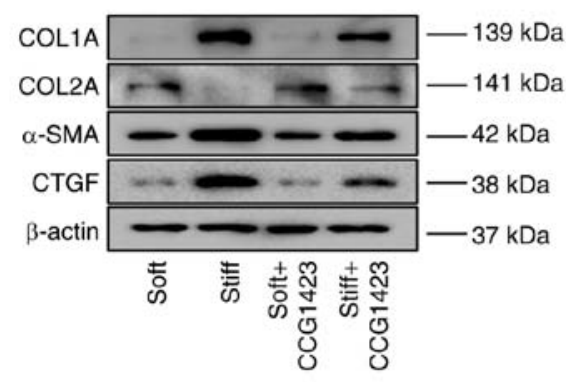

B

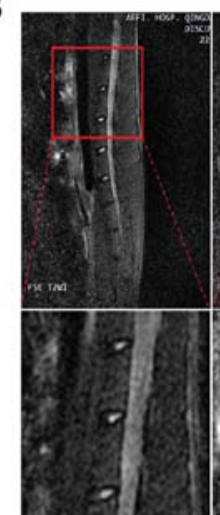

Pre-Model

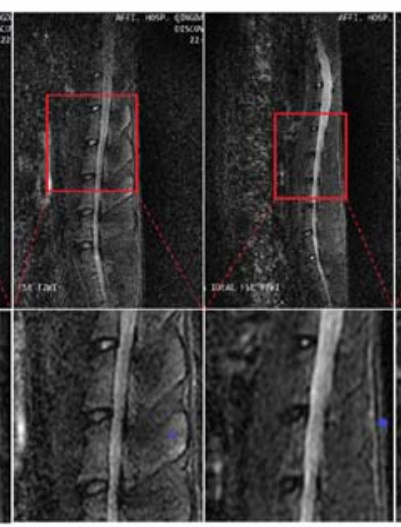

Control
Model

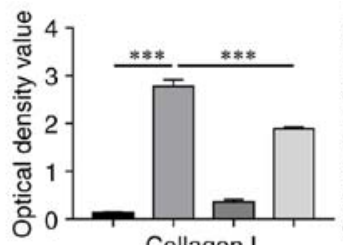

Collagen I

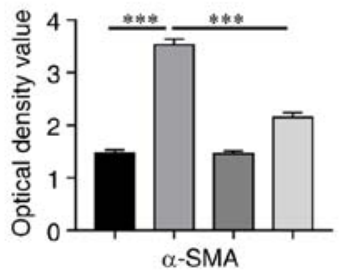

$\alpha-\mathrm{SMA}$
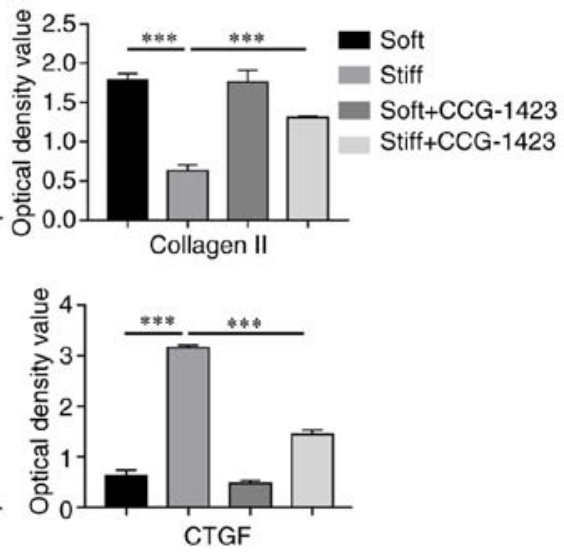

Figure 4. Fibrotic protein expression of NPCs and rat spine imaging. (A) Representative type I and II collagen, $\alpha$-SMA and CTGF western blots of NPCs treated in different conditions. Quantitation of protein expression as normalized to $\beta$-actin. $n=3$. (B) Representative MRI scans of the pre-modelling animals and the experimental groups at 3 months after drug injection. The selected region included L3/4, L4/5 and L5/6. The CCG-1423-treated group exhibited stronger signal intensities (red arrow) compared with those in the model and DMSO groups. Quantitative analysis of IVDD grade was performed by MRI according to the modified Thompson classification. ${ }^{*} \mathrm{P}<0.05,{ }^{* *} \mathrm{P}<0.01$ and ${ }^{* * *} \mathrm{P}<0.001$. NPCs, nucleus pulposus cells; MRI, magnetic resonance imaging; IVDD, intervertebral disc degeneration; $\alpha$-SMA, $\alpha$-smooth muscle actin; CTGF, connective tissue growth factor.

the cartilage end plate and AF, suggesting low exposure to mechanical stress. Degeneration of the IVDs in the IVDD model and DMSO groups was more severe compared with that in the control group. The qualitative signs were as follows: The volume of gelatinous NP was reduced; clefts were observed; the primary NP was replaced with disorganized collagen fibers; the density and volume of the NPCs low; and spindle-shaped fibroblast-like cells appeared. The indistinct boundary between the AF and NP indicated the formation of NP fibrosis (Fig. 5A). However, compared with IVDD model and DMSO group, fibrosis was relieved, and more even and regular cell distribution, relatively reduced disorganization of collagen fibers, and intact extracellular matrix alignment full of proteoglycan and water were identified in CCG-1423-treated IVDD group. The histological scores based on the H\&E staining results demonstrated that the model group exhibited higher degeneration scores compared with those in the control group, and the structure of the degenerative IVDD was improved by CCG-1423, but not by DMSO alone (Fig. 5C).

Long-term upright posture induces MRTF-A nuclear translocation, changes in F-actin expression and fibrosis biomarker expression, which is attenuated by CCG-1423 in the rat IVD. The present study further analyzed whether long-term mechanical stress altered MRTF-A distribution in rat NP samples. Immunohistochemical staining (Fig. 5B and C) revealed that sustained mechanical load provoked the nuclear accumulation of MRTF-A labeling in more NPCs compared with that in the normal control rats, which was consistent with the results demonstrated in vitro. In the CCG-1423 group, the number of NPCs exhibiting MRTF-A nuclear localization decreased compared with that in the model group, suggesting that orthotopic injection of CCG-1423 mitigated the mechanical load-induced MRTF-A nuclear translocation in initiated fibrotic NPs. These results suggested that MRTF-A nuclear-cytoplasmic shuttling may participate in NP fibrosis.

To determine whether the MRTF-A that accumulated in the nucleus was transferred from the cytoplasm, the expression of F-actin, a product of MRTF-A nuclear shuttling, was determined in the cytoplasm by immunofluorescence, and increased cytoplasmic staining intensity of F-actin was observed in the NP tissues of IVDD model rats compared with that in the control animals. In NP tissues treated with CCG-1423, cytoplasmic F-actin levels were reduced (Fig. 6). These results further confirmed the association between mechanical load stress and actin/MRTF-A signaling in the NP of rats.

The statistical analysis of the immunohistochemical staining results (Fig. 7) revealed that prolonged upright positioning led to heterogeneous trends of upregulated levels of type I collagen, 

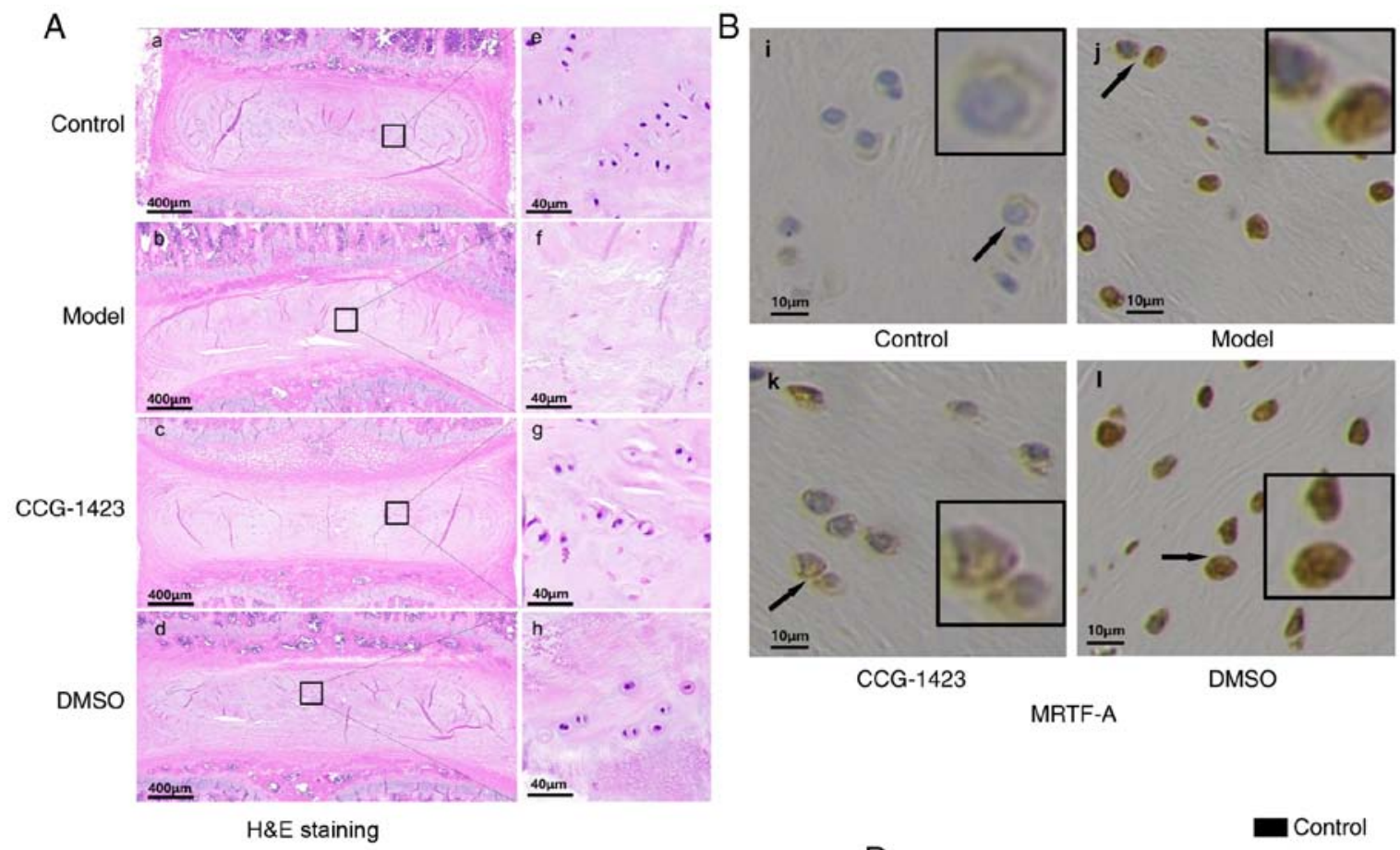

MRTF-A
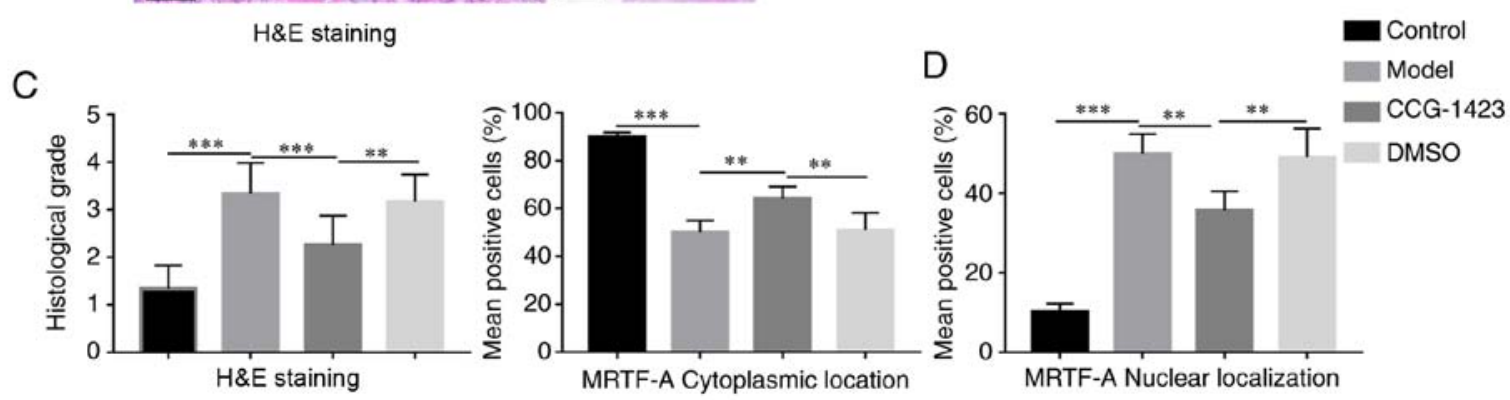

Figure 5. H\&E and immunohistochemical staining of intervertebral disc in the four groups of rats. (A-a-d) Overview of morphological changes in the discs of the four groups. (A-a) In the control group, the intervertebral altitude was reserved; the NP was highly hydrated; the boundary between the AF and the NP was distinct. (A-b and d) In the (A-b) model and (A-d) DMSO groups, the intervertebral space was narrow, and the boundary between the AF and the NP became indistinct. (A-c) In the CCG-1423-treated group, a reserved boundary was observed between the AF and the atrophic NP. (A-e-h) Magnified images of selected regions of the NP. (A-e) In the control group, the NP was highly cellular with notochordal and chondrocyte-like cells. (A-f and h) In the (A-f) model and (A-e) DMSO groups, the cell density was low; spindle-shaped fibroblast-like cells and filamentous collagen fibers with clefts were observed. (A-g) Low levels of hydration and cluster-distributed cells were observed in the CCG-1423-treated group, and clefts were rare. (B) Subcellular expression of MRTF-A in various disc tissues was detected by the immunohistochemical staining. Regions indicated by black arrows are magnified in black-bordered images. (C) The degree of IVDD in the model and DMSO groups was increased compared with that in the control group. Rats in the CCG-1423 group exhibited relatively a lower grade of IVDD compared with the model and DMSO groups. (D) Quantitative analysis of the subcellular localization of MRTF-A in the four groups based on the results of immunohistochemical staining. $n \geq 3 .{ }^{* *} \mathrm{P}<0.01$ and ${ }^{* * * *} \mathrm{P}<0.001$. IVDD, intervertebral disc degeneration; AF, annulus fibrosus; NP, nucleus pulposus; MRTF-A, myocardin-related transcription factor A.

CTGF and $\alpha$-SMA expression in the model group compared with those in the control group. The modelling-enhanced immunoreactivity was downregulated by CCG-1423 injection to varying degrees. These results suggested that type I collagen, CTGF and $\alpha$-SMA may serve important roles in the process of NP fibrosis in IVDD and validated the positive effects of CCG-1423 on fibrosis in a rat model of IVDD.

\section{Discussion}

NP fibrosis is a key complication of IVDD for which there is currently no available treatment $(31,32)$. Numerous in vivo studies in other organ systems have demonstrated the role of mechanoregulation in fibrosis initiation, ECM remodeling and subsequent tissue stiffening $(33,34)$.

It has been established in vitro that the Rho signaling pathway serves multiple functions ranging from cellular mechanosensing and motility to signal transduction and transcriptional regulation (35), and the Rho signaling pathway is a key pathway in the fibrosis of numerous types of solid organs at the organism level $(15,36)$. In light of the essential roles of Rho GTPases in cellular activity, the present study focused on a classic signaling pathway, namely, the mechanical force-stimulated Rho GTPase cascade, followed by actin filament recombination and pivotal MRTF-A nuclear translocation, which contributes to fibrotic gene expression; thus, this pathway links external stress to MRTF-A-mediated transcriptional activation and subsequent $\alpha$-SMA, type I collagen and CTGF expression, providing evidence for a mechanical force-induced transcriptional response that may contribute to the development of NP fibrosis.

Comprehensive research findings have indicated that multiple regimens of mechanosensing may interconnect to form a synergistic mechanosensing system upstream of MRTF-A; 


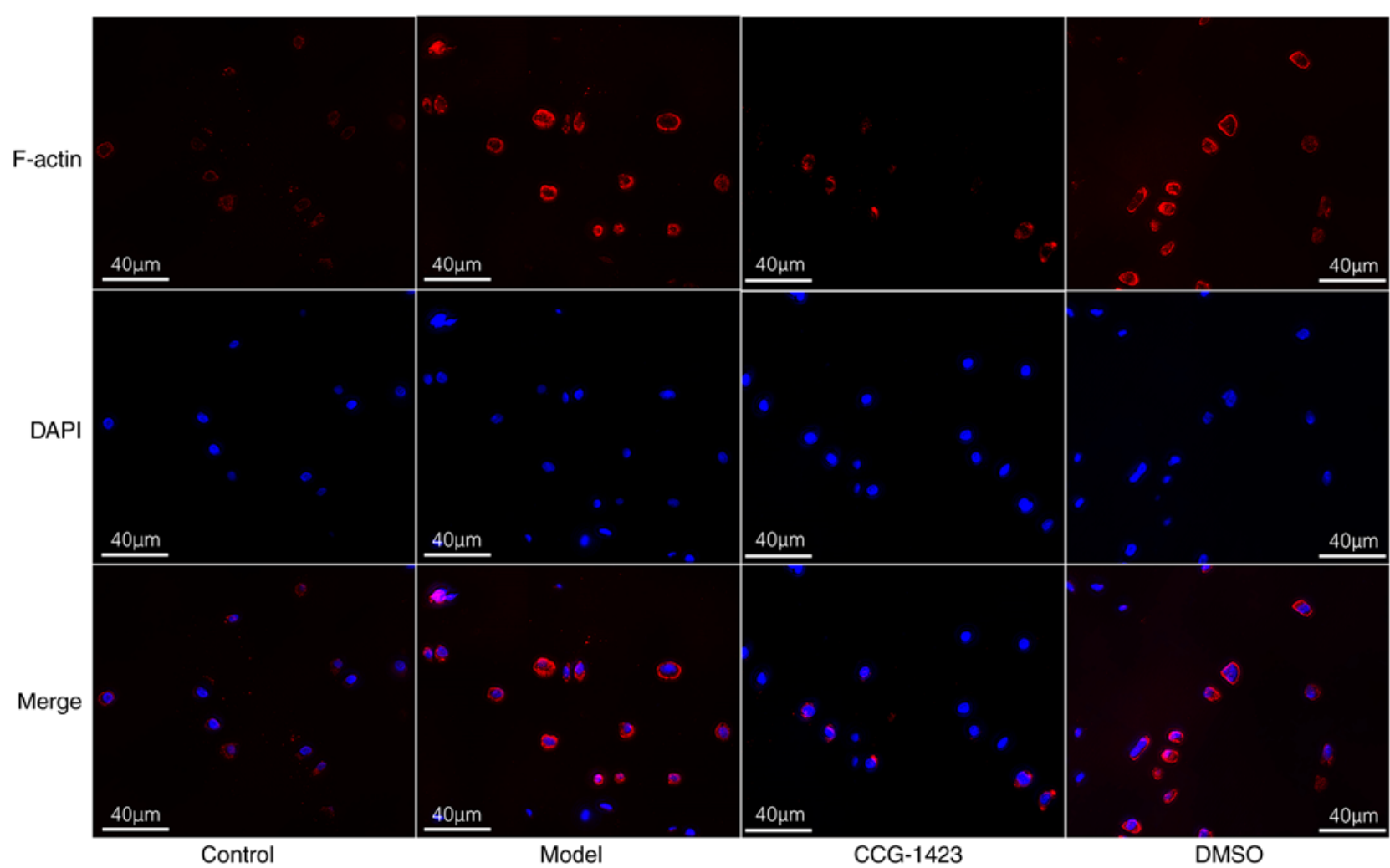

Figure 6. Representative images of F-actin immunostaining in the NP tissues from rats in the four groups. Intense signals were observed in the cytoplasm of NP cells of rats in the model and DMSO groups; F-actin staining was less intense in NP cells of rats that received CCG-1423 treatment and those of intact control animals. NP, nucleus pulposus.

these studies have demonstrated that Rho signaling includes a variety of intracellular molecules, such as Rho family small $\mathrm{G}$ protein subfamilies RhoA, Rac1, RhoC and Ras, and their downstream target Rho kinase, which are important regulators of the response to mechanical stress (37-39). For example, CTGF is a hallmark of fibrotic diseases, is upregulated in numerous pathological processes involving mechanically challenged organs, and promotes ECM accumulation and, notably, macromolecule synthesis (i.e., type I collagen) (40). Blomme et al (41) silenced RhoA, RhoC and Racl expression using small interfering RNAs in valvular interstitial cells and reported that Rac1 and RhoA were involved in regulating the basal level of CTGF, whereas RhoC potentially participated in the mechanical signaling-induced upregulation of CTGF gene expression. Therefore, the identification of the systematic mechanism in NP tissue is complicated. However, it has been demonstrated that the type I collagen, $\alpha$-SMA and CTGF gene transcription levels are directly mediated by cooperation between SRF and the strain-associated nuclear translocation of MRTF-A (42-44). Thus, the actin treadmill-dependent nuclear accumulation of MRTF-A appears to be a key mediator of the upstream signals in the progression of fibrogenesis.

Since the Rho signaling pathway is involved in various pathological processes in addition to fibrosis, such as the cell cycle, morphology and motility along with transcription regulation and reactive oxygen species production $(45,46)$, the multiple effects of the Rho signaling pathway have limited selective drug discovery efforts. RhoA inhibitors, which alter cytoskeletal dynamics, such as Y-27632, have limited clinical utility due to their potential side effects and weak efficacy (47). MRTF-A is sequestered in the cytoplasm by preferentially forming a complex with G-actin, which significantly attenuates the interaction between MRTF-A and importin $\alpha / \beta 1$, a regulator of the nuclear import of MRTF family members (48). In response to mechanical force, the activated Rho signaling pathway induces rapid depletion of the $\mathrm{G}$-actin pool, thus increasing the binding of G-actin-free MRTF-A to importin $\alpha / \beta 1$ and its nuclear localization (49). External force-reduced G-actin monomers in the cytoplasm assembly promote the accumulation of F-actin subjacent to the plasma membrane (50). Currently, pharmacological development has shifted from focusing on the upstream locus inhibition to targeting fibrotic transcription activation (MRTF-A/SRF) (51). Studies have identified that CCG-1423 acts as an inhibitor of the binding between MRTF-A and importin $\alpha / \beta 1$ protein $(49,52)$ due to the $\mathrm{N}$-terminal basic domain of G-actin-free MRTF-A, which functions as a nuclear localization signal (17). Therefore, the present study investigated MRTF-A nuclear-cytoplasmic trafficking downstream of the Rho signaling pathway and demonstrated it to be a potential clinical target for the treatment of mechanical stimulus-induced NP fibrogenesis. In addition, the beneficial antifibrotic efficacy of CCG-1423 was assessed both in vitro and in vivo by the functional inhibition of MRTF-A nuclear localization.

The present study used two approaches to test the role of stress-induced MRTF-A translocation and NP fibrosis in the process of IVDD. First, since PAG substrates with different stiffness (2.9 and $41.7 \mathrm{kPa}$ in the present study) effectively mimic various magnitudes of static tension sustained by NP cells from the mechanical environment $(53,54)$, the existence of this pathway in NPC was assessed in vitro, and the present study 


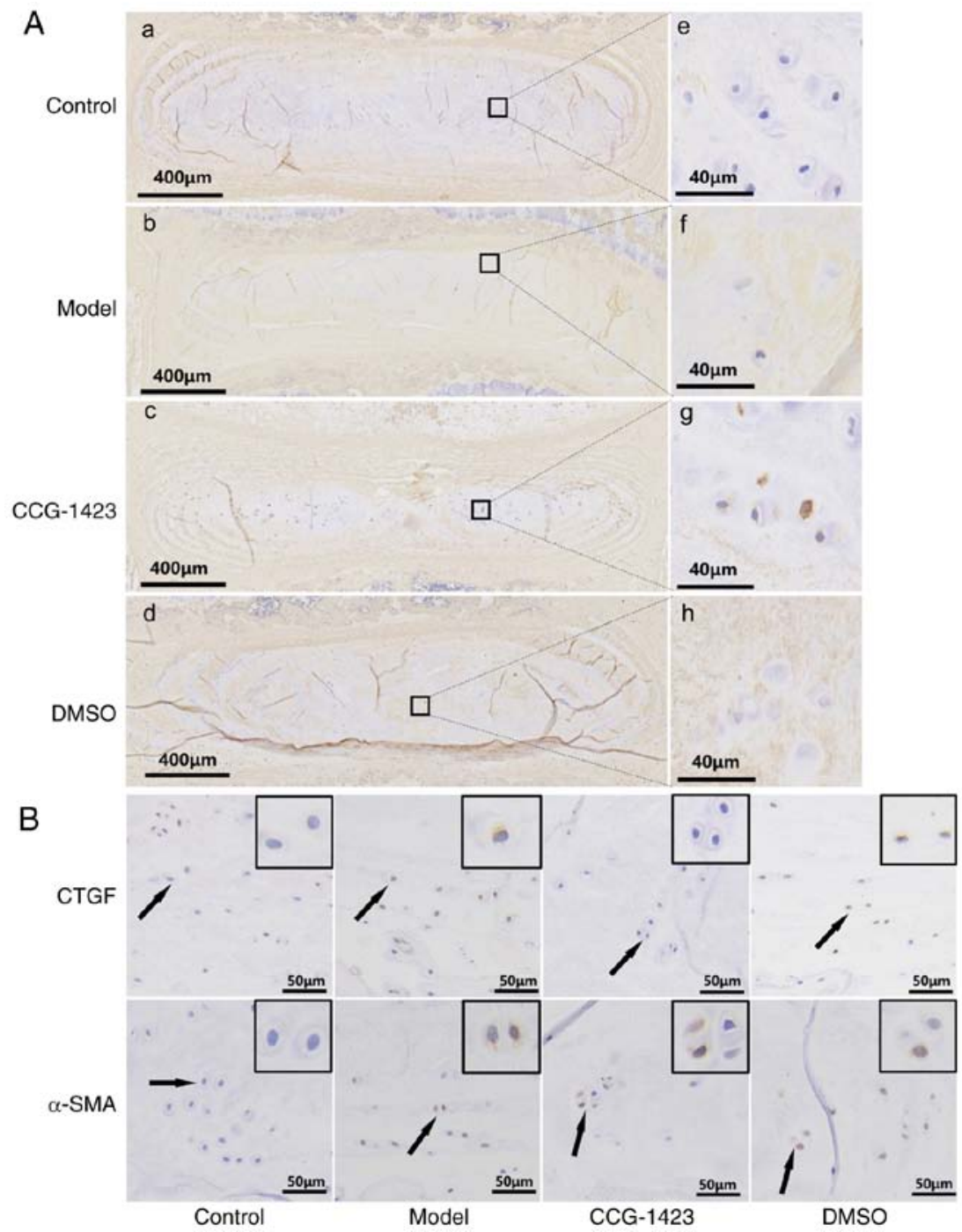

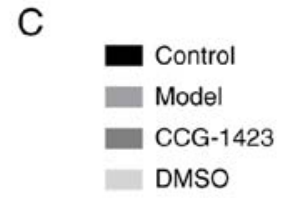
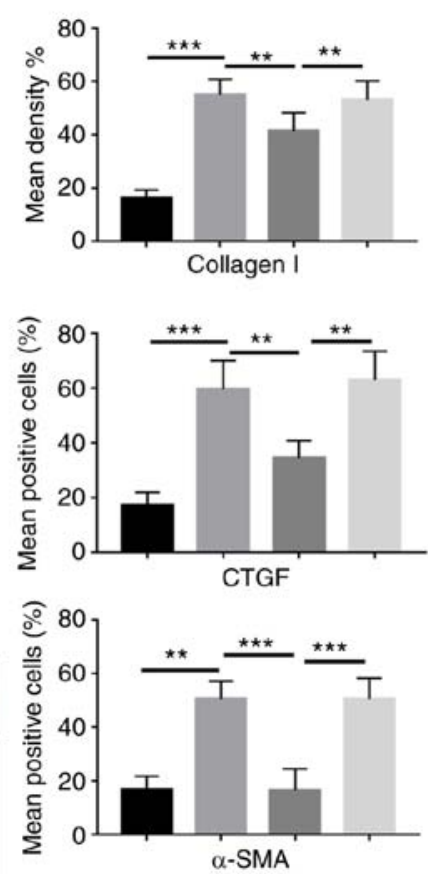

Figure 7. Inhibition of myocardin-related transcription factor A translocation alleviates the NP fibrosis phenotype in vivo. (A) Overview and magnified images of immunohistochemical staining for type I collagen in the intravertebral discs of rats in the four groups. (A-a and e) In the control group, type I collagen (brown) was mainly present around the NP, and most normal NP (blue) was retained. (A-b, f, $d$ and h) In the model and DMSO groups, the expression of type I collagen covered a large area of NP, and positive staining was detected in the intercellular substance of NP cells. (A-c and g) The area of NP with positive type I collagen staining was smaller following treatment with CCG-1423 compared with that in the model and DMSO groups, and the boundary between the AF and the NP remained distinct. (B) Following 6 months of upright posture, upregulated expression levels of CTGF and $\alpha$-SMA were observed in the NP of bipedal rats compared with those in the control group, which were reduced by CCG-1423 treatment. (C) Quantitative analysis of the positive area of type I collagen, CTGF and $\alpha$-SMA in the four groups based on the immunohistochemical staining. ${ }^{* *} \mathrm{P}<0.01$ and ${ }^{* * *} \mathrm{P}<0.001$. NP, nucleus pulposus; AF, annulus fibrosus; SMA, $\alpha$-smooth muscle actin; CTGF, connective tissue growth factor.

further examined whether NPCs cultured on soft and stiff matrix substrates expressed different fibrogenic proteins, and whether interference with MRTF-A signaling may alter the production of mechanically induced cytokines. Furthermore, using a brachial plexus rhizotomy approach, a bipedal rat model was established to imitate the upright posture of humans and exacerbate the transmitted force input to the IVD. Th present study tested whether long-term upright posture induced rat lumbar disc NP fibrogenesis associated with the upregulation of CTGF, $\alpha$-SMA and type I collagen; the results demonstrated that the disruption of MRTF-A postponed the fibrogenic process in the NP.

The NP tissue is the inner gel-like portion of the IVD that it is composed primarily of small cartilage-like NPCs stemming from notochord cells and other ECM components, and large aggregating proteoglycans loosely held together by a network of type II collagen and elastin fibers (55). The influx of water molecules to the NP is created by osmotic pressure, which is maintained by sulfated glycosaminoglycans in the NP system and provides the elasticity required for the NP to keep the body stable against compressive loads (56). Previous studies have reported that type II collagen, the levels of which gradually decrease from the inner NP to the outer AP, is replaced by high expression levels of type I collagen in degenerative discs $(57,58)$. Consistently, type II collagen protein expression levels were decreased in NPCs cultured on a stiff substrate in the present study, which was opposite to the changes observed in type I collagen expression. These pathological alterations may be responsible for the NP degeneration and dehydration that occurred in the bipedal rat model, as observed by T2-weighted MR imaging. These results suggested that the IVDD models were successfully established, which laid the foundation for subsequent experiments. 
The nuclear translocation of MRTF-A in response to high mechanical force has been identified in primary studies with various human cell types, such as human colonic fibroblasts and intestinal cells (59-61). Consistent with previous data in the fibrosis of other organs, the present study first demonstrated that mechanical stiffness of the culture substrate affected the nucleocytoplasmic shuttling of MRTF-A in rat NPCs in vitro. Consistent with a previous study (62), the results of the present study demonstrated upregulated expression levels of fibrotic markers of the ECM, such as $\alpha$-SMA, type I collagen and CTGF, in the NP samples of a bipedal rat IVDD model, and the levels of these markers were significantly attenuated in the CCG-1423-treated animals. These results suggested the involvement of MRTF-A nuclear-cytoplasmic trafficking in the regulation of the expression of a series of fibrosis-associated proteins induced by excessive external stress.

In the present study, CCG-1423 treatment partly abolished the matrix stiffness-dependent upregulation of fibrotic protein expression levels in vitro. Notably, no further suppression of fibrotic protein expression levels was observed in NPCs co-treated with physiological stiffness and inhibitors in the stiff + CCG-1423 group, and these levels may represent the basal level. These results were consistent with a previous study, which demonstrated the basal expression of CTGF observed in a static condition (41). In addition, CCG-1423 treatment had no effect on cell viability, indicating that the downregulation of fibrotic protein expression in NPCs was not associated with the cytotoxicity of CCG-1423. Finally, F-actin was studied to determine the role of MRTF-A in regulating the differentiation fate of NPCs by mediating the actin dynamics. The results demonstrated that MRTF-A inhibition significantly decreased the intensity of F-actin staining; a similar inhibitory effect on F-actin has been recently demonstrated in human adipose stem cells treated with CCG-1423 (63). These results further suggested that MRTF-A together with fibrotic proteins participate in the fibrogenesis initiated by mechanical overload by coupling actin. In conclusion, the results of the present study suggested that strain-derived MRTF-A translocation-regulated fibrotic protein expression may be a key genetic switch in the development of NP fibrosis, and provide a theoretical basis for targeting this signaling pathway as an effective therapeutic approach. In a model of bipedal rats, the therapeutic administration of CCG-1423 significantly limited the extent of NP fibrosis. Thus, these findings may aid in the formulation of mechanotransduction-based therapies or the identification of drug targets for treating IVDD at the molecular level to prevent or reverse the deleterious effects of mechanical overload.

\section{Acknowledgements}

Not applicable.

\section{Funding}

This work was supported by the National Natural Science Foundation of China (grant nos. 81672200 and 81871804) and the National Key Research and Development Project of China (grant no. 2019YFC0121400).

\section{Availability of data and materials}

The datasets used and/or analyzed during the current study are available from the corresponding author on reasonable request.

\section{Authors' contributions}

MK and YZ performed the experiments, analyzed data and drafted the manuscript. MS performed some of the experiments. MK, YZ and MS confirm the authenticity of all the raw data. WC and CG contributed to the data analysis and interpretation. JZ, SH and QT performed the in vivo experiments, data collection and analysis. XM designed the study, supervised data analysis and revised the manuscript. All authors read and approved the final manuscript.

\section{Ethics approval and consent to participate}

The study was approved by the Laboratory Animal Care and Use Committee of the Affiliated Hospital of Qingdao University (Qing'dao, China; approval no. QYFYWZLL 26037).

\section{Patient consent for publication}

Not applicable.

\section{Competing interests}

The authors declare that they have no competing interests.

\section{References}

1. Fatoye F, Gebrye T and Odeyemi I: Real-world incidence and prevalence of low back pain using routinely collected data. Rheumatol Int 39: 619-626, 2019.

2. GBD 2017 Disease and Injury Incidence and Prevalence Collaborators: Global, regional, and national incidence, prevalence, and years lived with disability for 354 diseases and injuries for 195 countries and territories, 1990-2017: A systematic analysis for the Global Burden of Disease Study 2017. Lancet 392: 1789-1858, 2018

3. Kleinman N, Patel AA, Benson C, Macario A, Kim M and Biondi DM: Economic burden of back and neck pain: Effect of a neuropathic component. Popul Health Manag 17: 224-232, 2014.

4. Neidlinger-Wilke C, Galbusera F, Pratsinis H, Mavrogonatou E, Mietsch A, Kletsas D and Wilke HJ: Mechanical loading of the intervertebral disc: From the macroscopic to the cellular level. Eur Spine J 23 (Suppl 3): S333-S343, 2014.

5. Setton LA and Chen J: Mechanobiology of the intervertebral disc and relevance to disc degeneration. J Bone Joint Surg Am 88 (Suppl 2): S52-S57, 2006.

6. Pengb Y and Lv FJ: Fibrosis in intervertebral disc degeneration: Knowledge and gaps. Austin J Orthopade Rheumatol 1: 3, 2014.

7. Yee A, Lam MP, Tam V, Chan WC, Chu IK, Cheah KS, Cheung KM and Chan D: Fibrotic-like changes in degenerate human intervertebral discs revealed by quantitative proteomic analysis. Osteoarthritis Cartilage 24: 503-513, 2016.

8. Nanthakumar CB, Hatley RJ, Lemma S, Gauldie J, Marshall RP and Macdonald SJ: Dissecting fibrosis: Therapeutic insights from the small-molecule toolbox. Nat Rev Drug Discov 14: 693-720, 2015.

9. Olson EN and Nordheim A: Linking actin dynamics and gene transcription to drive cellular motile functions. Nat Rev Mol Cell Biol 11: 353-365, 2010.

10. Marinissen MJ, Chiariello M, Tanos T, Bernard O, Narumiya S and Gutkind JS: The small GTP-binding protein RhoA regulates c-jun by a ROCK-JNK signaling axis. Mol Cell 14: 29-41, 2004. 
11. Liu F, Mih JD, Shea BS, Kho AT, Sharif AS, Tager AM and Tschumperlin DJ: Feedback amplification of fibrosis through matrix stiffening and COX-2 suppression. J Cell Biol 190: 693-706, 2010

12. Shimizu Y, Dobashi K, Iizuka K, Horie T, Suzuki K, Tukagoshi H, Nakazawa T, Nakazato Y and Mori M: Contribution of small GTPase Rho and its target protein rock in a murine model of lung fibrosis. Am J Respir Crit Care Med 163: 210-217, 2001.

13. Satoh S, Ueda Y, Koyanagi M, Kadokami T, Sugano M, Yoshikawa Y and Makino N: Chronic inhibition of Rho kinase blunts the process of left ventricular hypertrophy leading to cardiac contractile dysfunction in hypertension-induced heart failure. J Mol Cell Cardiol 35: 59-70, 2003.

14. Bei Y,Hua-Huy T, Nicco C, Duong-Quy S, Le-Dong NN, Tiev KP, Chéreau C, Batteux F and Dinh-Xuan AT: RhoA/Rho-kinase activation promotes lung fibrosis in an animal model of systemic sclerosis. Exp Lung Res 42: 44-55, 2016.

15. Okumura N, Koizumi N, Ueno M, Sakamoto Y, Takahashi H, Hirata K, Torii R, Hamuro J and Kinoshita S: Enhancement of corneal endothelium wound healing by Rho-associated kinase (ROCK) inhibitor eye drops. Br J Ophthalmol 95: 1006-1009, 2011

16. Miralles F, Posern G, Zaromytidou AI and Treisman R: Actin dynamics control SRF activity by regulation of its coactivator MAL. Cell 113: 329-342, 2003.

17. Hayashi K, Watanabe B, Nakagawa Y, Minami S and Morita $\mathrm{T}$ : RPEL proteins are the molecular targets for CCG-1423, an inhibitor of Rho signaling. PLoS One 9: e89016, 2014

18. Hiyama A, Arai F, Sakai D, Yokoyama K and Mochida J: The effects of oxygen tension and antiaging factor Klotho on Wn signaling in nucleus pulposus cells. Arthritis Res Ther 14: R105, 2012.

19. Pelham RJ Jr and Wang Yl: Cell locomotion and focal adhesions are regulated by substrate flexibility. Proc Natl Acad Sci USA 94: 13661-13665, 1997.

20. Tse JR and Engler AJ: Preparation of hydrogel substrates with tunable mechanical properties. Curr Protoc Cell Biol Chapter 10: Unit 10.16, 2010

21. Gilchrist CL, Darling EM, Chen J and Setton LA: Extracellular matrix ligand and stiffness modulate immature nucleus pulposus cell-cell interactions. PLoS One 6: e27170, 2011.

22. Wallace MA, Della Gatta PA, Ahmad Mir B, Kowalski GM, Kloehn J, McConville MJ, Russell AP and Lamon S: Overexpression of striated muscle activator of Rho signaling (STARS) increases C2C12 skeletal muscle cell differentiation. Front Physiol 7: 7, 2016.

23. Esnault C, Stewart A, Gualdrini F, East P, Horswell S, Matthews N and Treisman R: Rho-actin signaling to the MRTF coactivators dominates the immediate transcriptional response to serum in fibroblasts. Genes Dev 28: 943-958, 2014

24. Xiao J, Qiu GX, Wu ZH, Xu J, Wang T, Wang YP and Weng XS An improved operation approach for bipedal rat model construction. Zhonghua Yi Xue Za Zhi 86: 2781-2785, 2006 (In Chinese)

25. Liang X, Shen H, Shi WD, Ren S, Jiang W, Liu H, Yang P, Sun ZY, Lin J and Yang HL: Effect of axial vertical vibration on degeneration of lumbar intervertebral discs in modified bipedal rats: An in-vivo study. Asian Pac J Trop Med 10: 714-717, 2017.

26. Thompson JP, Pearce RH, Schechter MT, Adams ME, Tsang IK and Bishop PB: Preliminary evaluation of a scheme for grading the gross morphology of the human intervertebral disc. Spine (Phila Pa 1976) 15: 411-415, 1990

27. Ge J, Cheng X, Yuan C, Qian J, Wu C, Cao C, Yang H, Zhou F and Zou J: Syndecan-4 is a novel therapeutic target for intervertebral disc degeneration via suppressing JNK/p53 pathway. Int J Biol Sci 16: 766-776, 2020

28. Yang F, Leung VY, Luk KD, Chan D and Cheung KM Injury-induced sequential transformation of notochordal nucleus pulposus to chondrogenic and fibrocartilaginous phenotype in the mouse. J Pathol 218: 113-121, 2009.

29. Tam V, Chan WCW, Leung VYL, Cheah KSE, Cheung KMC, Sakai D, McCann MR, Bedore J, Séguin CA and Chan D Histological and reference system for the analysis of mouse intervertebral disc. J Orthop Res 36: 233-243, 2018.

30. Manohar M, Kandikattu HK, Verma AK and Mishra A: IL-15 regulates fibrosis and inflammation in a mouse model of chronic pancreatitis. Am J Physiol Gastrointest Liver Physiol 315: G954-G965, 2018.

31. Cui L, Wei H, Li ZM, Dong XB and Wang PY: TGF- $\beta 1$ aggravates degenerative nucleus pulposus cells inflammation and fibrosis through the upregulation of angiopoietin-like protein 2 expression. Eur Rev Med Pharmacol Sci 24: 12025-12033, 2020.
32. Meng X, Zhuang L, Wang J, Liu Z, Wang Y, Xiao D and Zhang X: Hypoxia-inducible factor (HIF)-1alpha knockout accelerates intervertebral disc degeneration in mice. Int J Clin Exp Pathol 11: 548-557, 2018

33. Eckes B, Nischt R and Krieg T: Cell-matrix interactions in dermal repair and scarring. Fibrogenesis Tissue Repair 3: 4, 2010.

34. Hinz B: The myofibroblast: Paradigm for a mechanically active cell. J Biomech 43: 146-155, 2010.

35. Amano M, Nakayama M and Kaibuchi K: Rho-kinase/ROCK: A key regulator of the cytoskeleton and cell polarity. Cytoskeleton (Hoboken) 67: 545-554, 2010.

36. Bond JE, Kokosis G, Ren L, Selim MA, Bergeron A and Levinson H: Wound contraction is attenuated by fasudil inhibition of Rho-associated kinase. Plast Reconstr Surg 128 $438 \mathrm{e}-450 \mathrm{e}, 2011$.

37. Brown JH, Del Re DP and Sussman MA: The Rac and Rho hall of fame: A decade of hypertrophic signaling hits. Circ Res 98: 730-742, 2006.

38. Holle AW and Engler AJ: More than a feeling: Discovering, understanding, and influencing mechanosensing pathways. Curr Opin Biotechnol 22: 648-654, 2011.

39. Geneste O, Copeland JW and Treisman R: LIM kinase and Diaphanous cooperate to regulate serum response factor and actin dynamics. J Cell Biol 157: 831-838, 2002.

40. Leask A, Parapuram SK, Shi-Wen X and Abraham DJ: Connective tissue growth factor $(\mathrm{CTGF}, \mathrm{CCN} 2)$ gene regulation: A potent clinical bio-marker of fibroproliferative disease? J Cell Commun Signal 3: 89-94, 2009.

41. Blomme B, Deroanne C, Hulin A, Lambert C, Defraigne JO, Nusgens B, Radermecker M and Colige A: Mechanical strain induces a pro-fibrotic phenotype in human mitral valvular interstitial cells through RhoC/ROCK/MRTF-A and Erk1/2 signaling pathways. J Mol Cell Cardiol 135: 149-159, 2019.

42. Johnson LA, Rodansky ES, Haak AJ, Larsen SD, Neubig RR and Higgins PD: Novel Rho/MRTF/SRF inhibitors block matrix-stiffness and TGF- $\beta$-induced fibrogenesis in human colonic myofibroblasts. Inflamm Bowel Dis 20: 154-165, 2014.

43. Shiwen X, Stratton R, Nikitorowicz-Buniak J, Ahmed-Abdi B Ponticos M, Denton C, Abraham D, Takahashi A, Suki B, Layne MD, et al: A role of myocardin related transcription factor-A (MRTF-A) in scleroderma related fibrosis. PLoS One 10: e0126015, 2015 .

44. Shao J, Xu H, Wu X and $\mathrm{Xu} \mathrm{Y}$ : Epigenetic activation of CTGF transcription by high glucose in renal tubular epithelial cells is mediated by myocardin-related transcription factor A. Cell Tissue Res 379: 549-559, 2020.

45. Arthur WT, Noren NK and Burridge K: Regulation of Rho family GTPases by cell-cell and cell-matrix adhesion. Biol Res 35: 239-246, 2002.

46. Kim JG, Islam R, Cho JY, Jeong H, Cap KC, Park Y, Hossain AJ and Park JB: Regulation of RhoA GTPase and various transcription factors in the RhoA pathway. J Cell Physiol 233: 6381-6392, 2018.

47. Takahara A, Sugiyama A, Satoh Y, Yoneyama M and Hashimoto K: Cardiovascular effects of Y-27632, a selective Rho-associated kinase inhibitor, assessed in the halothane-anesthetized canine model. Eur J Pharmacol 460: 51-57, 2003.

48. Hinson JS, Medlin MD, Lockman K, Taylor JM and Mack CP: Smooth muscle cell-specific transcription is regulated by nuclear localization of the myocardin-related transcription factors. Am J Physiol Heart Circ Physiol 292: H1170-1180, 2007.

49. Pawłowski R, Rajakylä EK, Vartiainen MK and Treisman R: An actin-regulated importin $\alpha / \beta$-dependent extended bipartite NLS directs nuclear import of MRTF-A. EMBO J 29: 3448-3458, 2010.

50. Dai J, Qin L, Chen Y, Wang H, Lin G, Li X, Liao H and Fang H: Matrix stiffness regulates epithelial-mesenchymal transition via cytoskeletal remodeling and MRTF-A translocation in osteosarcoma cells. J Mech Behav Biomed Mater 90: 226-238, 2019.

51. Hahmann C and Schroeter T: Rho-kinase inhibitors as therapeutics: From pan inhibition to isoform selectivity. Cell Mol Life Sci 67: 171-177, 2010.

52. Nakamura S, Hayashi K, Iwasaki K, Fujioka T, Egusa H, Yatani H and Sobue K: Nuclear import mechanism for myocardin family members and their correlation with vascular smooth muscle cell phenotype. J Biol Chem 285: 37314-37323, 2010.

53. Zhang YH, Zhao CQ, Jiang LS and Dai LY: Substrate stiffness regulates apoptosis and the mRNA expression of extracellular matrix regulatory genes in the rat annular cells. Matrix Biol 30: 135-144, 2011. 
54. Hoffman BD, Grashoff C and Schwartz MA: Dynamic molecular processes mediate cellular mechanotransduction. Nature 475: 316-323, 2011.

55. Waxenbaum JA, Reddy V and Futterman B: Anatomy, Back, Intervertebral Discs. In: StatPearls. StatPearls Publishing. Copyright $^{\oplus}$ 2020, StatPearls Publishing LLC, Treasure Island, FL, 2020.

56. Sivan SS, Roberts S, Urban JP, Menage J, Bramhill J, Campbell D, Franklin VJ, Lydon F, Merkher Y, Maroudas A and Tighe BJ: Injectable hydrogels with high fixed charge density and swelling pressure for nucleus pulposus repair: Biomimetic glycosaminoglycan analogues. Acta Biomater 10: 1124-1133, 2014.

57. Chelberg MK, Banks GM, Geiger DF and Oegema TR Jr: Identification of heterogeneous cell populations in normal human intervertebral disc. J Anat 186: 43-53, 1995.

58. Zhang YG, Sun ZM, Liu JT, Wang SJ, Ren FL and Guo X: Features of intervertebral disc degeneration in rat's aging process. J Zhejiang Univ Sci B 10: 522-527, 2009.

59. Huang X, Yang N, Fiore VF, Barker TH, Sun Y, Morris SW Ding Q, Thannickal VJ and Zhou Y: Matrix stiffness-induced myofibroblast differentiation is mediated by intrinsic mechanotransduction. Am J Respir Cell Mol Biol 47: 340-348, 2012.
60. Zhao XH, Laschinger C, Arora P, Szászi K, Kapus A and McCulloch CA: Force activates smooth muscle alpha-actin promoter activity through the Rho signaling pathway. Cell Sci 120: 1801-1809, 2007.

61. Johnson LA, Rodansky ES, Sauder KL, Horowitz JC, Mih JD, Tschumperlin DJ and Higgins PD: Matrix stiffness corresponding to strictured bowel induces a fibrogenic response in human colonic fibroblasts. Inflamm Bowel Dis 19: 891-903, 2013.

62. Zheng L, Qin J, Sun L, Gui L, Zhang C, Huang Y, Deng W, Huang A, Sun D and Luo M: Intrahepatic upregulation of MRTF-A signaling contributes to increased hepatic vascular resistance in cirrhotic rats with portal hypertension. Clin Res Hepatol Gastroenterol 41: 303-310, 2017.

63. Hyväri L, Vanhatupa S, Halonen HT, Kääriäinen $M$ and Miettinen S: Myocardin-related transcription factor A (MRTF-A) regulates the balance between adipogenesis and osteogenesis of human adipose stem cells. Stem Cells Int 2020: 8853541, 2020. 